\title{
Utilização do Aparelho de Oscilação Oral de Alta Freqüência com Ventilador Mecânico*
}

\author{
Utilization of a High Frequency Oral Oscillatory \\ Device with Mechanical Ventilation
}

Rachel Maria da Silva Bezerra Fitipaldi', Carlos Alberto Caetano Azeredo

\section{RESUMO}

JUSTIFICATIVA E OBJETIVOS: A terapia de oscilação oral de alta freqüência (HOAF) é realizada através de aparelho constituído de material plástico em forma de cachimbo. Ele possui uma esfera metálica que, oscila durante a expiração, gerando pressão positiva expiratória oscilante. A proposta deste estudo foi verificar o desempenho do aparelho de HOAF nacional (Shaker, NCS, São Paulo) em relação à freqüência de oscilação e a pressão expiratória com a variação do fluxo aéreo e da inclinação.

MÉTODO: O aparelho foi conectado a um circuito que consistia de um pneumotacógrafo e de um ventilador. Ele teve o fluxo variado e durante esta variação a pressão expiratória e a freqüência de oscilação do apareIho eram mensurados em angulações que variavam de $+40^{\circ}$ à $-40^{\circ}$.

RESULTADOS: Foi encontrada correlação significativa entre o fluxo e a pressão expiratória em cada nível de inclinação. Uma maior freqüência de oscilação e pressão foi constatada nas angulações positivas com os fluxos maiores (50 e $60 \mathrm{~L} / \mathrm{min}$ ).

CONCLUSÕES: O aparelho de oscilação oral de alta freqüência pode ser utilizado como auxiliar na terapia de desobstrução brônquica durante a venti-

1. Fisioterapeuta- Mestre em Ciências da Motricidade Humana-UCB

2. Fisioterapeuta- Especialista em Fisioterapia Respiratória- Sobrafir

${ }^{*}$ Recebido do Centro de Estudos e Pesquisas Avançadas em Fisioterapia (CEPAF)

Apresentado em 11 de abril de 2005.

Aceito para publicação em 16 de fevereiro de 2006

Endereço para correspondência

Rachel Maria da Silva Bezerra Fitipaldi

Rua Marechal Marques Porto, 2/404 - Tijuca

20270-260 Rio de Janeiro, RJ

Fone: (021) 2254-0714

E-mail: rachelbezerra@aol.com lação mecânica.

Unitermos: fisioterapia, HOAF, mecânica, respiratória, ventilação.

\section{SUMMARY}

BACKGROUD AND OBJECTIVES: The high frequency oral oscillation therapy (HFOO) is carried through a plastic device which promotes the clearance of pulmonary sputum. This possesses a metallic sphere which, oscillates during the expiration, generating oscillatory positive expiratory pressure. The aim of this study was to verify the performance of the national device of HFOO (Shaker, NCS, São Paulo) in relation to frequency of oscillation and expiratory pressure with variation of flow and inclination.

METHODS: The device was imprisoned to a circuit that consisted of a pneumotacograph and a mechanical ventilator. This had the varied flow and during this variation the expiratory pressure and the frequency of oscillation of the device were measured in angulations which, varied of $+40^{\circ}$ to $-40^{\circ}$.

RESULTS: Significant correlation between flow and expiratory pressure in each level of inclination was found. A bigger frequency of oscillation and pressure was evidenced in the positive angulations with the biggest flows (50 and $60 \mathrm{~L} / \mathrm{min}$ ).

CONCLUSIONS: The high frequency oral oscillation device can be used as an assist for the air way clearance therapy during mechanical ventilation

Key Words: Chest, HFOO, mechanical, physiotherapy, ventilation

\section{INTRODUÇÃO}

A fisioterapia respiratória é essencial no tratamento de pacientes com acometimentos respiratórios agudos e crônicos, sendo a dificuldade em expectorar as secreções retidas o principal desafio. Nos últi- 
mos 40 anos o entendimento dos mecanismos de depuração das vias aéreas em situações normais e patológicas, tem aumentado ${ }^{1}$. Tem sido demonstrado, por exemplo, que a hipersecreção da mucosa predispõe a infecção brônquica recorrente e que a sua redução nas vias aéreas resulta em melhora do estado clínico ${ }^{2}$. Vários são os recursos fisioterapêuticos disponíveis nos mercados nacional e internacional desenvolvidos com objetivo de promover a limpeza das vias aéreas. Dentre estes se destaca o aparelho de oscilação oral de alta freqüência. O princípio de funcionamento deste aparelho é a expiração através de um orifício perfurado que produzirá a oscilação em alta freqüência de uma bola metálica $^{3}$. Este processo facilita a eliminação de secreção e saliva através da redução da viscoelasticidade ${ }^{4}$ e impedância mecânica do muco ${ }^{5}$. Outros benefícios desta terapia foram constatados como maior penetração de medicação broncodilatadora como resultado da mobilização do muco retido, a manutenção ou melhora da função pulmonar a longo prazo e maior estabilidade das unidades alveolares ao final da expiração6-8.

A assistência ao paciente ventilado mecanicamente é papel do terapeuta respiratório uma vez que diversas são as complicações pulmonares advindas deste processo. A principal delas seria o risco de infecção pulmonar, em parte devido ao desenvolvimento de bactérias na superfície interna e externa do tubo ${ }^{9,10}$. Uma das técnicas fisioterapêuticas implementadas porém, com pouco respaldo científico é a utilização de aparelhos de oscilação oral no ramo expiratório do ventilador mecânico.

Com o objetivo de verificar a efetividade desta técnica associada ao ventilador mecânico, foram avaliados em modelo in vitro os efeitos da variação do fluxo e da inclinação do aparelho em relação à freqüência de oscilação e a pressão expiratória gerada.

\section{MÉTODO}

O aparelho Shaker foi conectado ao ramo expiratório de um ventilador mecânico (Benett MA1) e a um pneumotacógrafo (Ventrak, Novametrix) para medida do fluxo, pressão e tempo. O sensor de pressão foi colocado próximo ao cone que ampara a bola metálica do aparelho por meio de uma perfuração realizada no seu fundo. No ramo inspiratório foi acoplado um pulmão de teste que, era manualmente desinsuflado. O modelo foi posteriormente, aficionado a um torno mecânico e as angulações do aparelho variadas com o auxílio de um goniômetro. $\mathrm{O}$ aparelho foi colocado em cinco angulações diferentes $\left(0^{\circ}, 30^{\circ}, 40^{\circ},-30^{\circ},-40^{\circ}\right)$. A linha horizontal foi considerada o ponto de partida para as variações na angulação do aparelho. Em cada angulação o fluxo foi variado três vezes $(40,50$ e $60 \mathrm{~L} / \mathrm{min})$. Os parâmetros de regulagem do ventilador mecânico foram freqüência respiratória: $10 \mathrm{irpm}$, nível ZEEP, VC: $1,200 \mathrm{~mL}$. A freqüência de oscilação e a pressão gerada durante a expiração foram determinadas através da contagem manual do gráfico fluxo versus tempo e pressão versus tempo, respectivamente. Para tanto foi utilizado um scaner (Canon 330dpi) para a digitalização e ampliação do gráfico.

Em cada ângulo avaliado $\left(0^{\circ}, 30^{\circ}, 40^{\circ},-30^{\circ}\right.$ e $\left.-40^{\circ}\right)$ as variáveis de interesse (pressão expiratória e freqüência de oscilação) foram promediadas em 10 ciclos. Em cada nível de inclinação, três fluxos diferentes $(40,50$ e $60 \mathrm{~L} / \mathrm{min}$ ) foram ajustados. A relação das variáveis (pressão e fluxo), com a inclinação do aparelho foi analisada, através de correlação (SPSS 10) e demonstrada em figuras.

\section{RESULTADOS}

Através do modelo experimental utilizado constatouse que a freqüência de oscilação do aparelho Shaker com os fluxos e angulações adotados variou de 9,33 a $16,66 \mathrm{~Hz}( \pm 13 \mathrm{~Hz}$, DP: 3,19). Com relação à pressão expiratória foram alcançados valores que variaram entre 11,33 e $18 \mathrm{cmH}_{2} \mathrm{O} \pm 14 \mathrm{cmH}_{2} \mathrm{O}$, DP: 2,88 (Tabela 1).

Tabela 1 - Valores da Pressão e a Freqüência nas Diferentes Angulações (média \pm DP)

\begin{tabular}{l|c|c}
\hline Angulação & Pressão & Freqüência \\
\hline$-40^{\circ}$ & $11,33 \pm 1,15$ & $9,33 \pm 0,57$ \\
$-30^{\circ}$ & $12 \pm 1$ & $10 \pm 0$ \\
$0^{\circ}$ & $14,66 \pm 0,57$ & $13,33 \pm 0,57$ \\
$30^{\circ}$ & $17 \pm 1$ & $16 \pm 1,73$ \\
$40^{\circ}$ & $18 \pm 1,73$ & $16,66 \pm 0,57$ \\
\hline
\end{tabular}

A figura 1 mostra a correlação entre a freqüência de oscilação e a angulação do aparelho em níveis de inclinação que variaram em $0^{\circ}, 30^{\circ}, 40^{\circ},-30^{\circ}$ e $-40^{\circ}$. Cada ponto representa a média de dez ciclos em cada nível de fluxo. Foi constatada uma forte correlação positiva entre a freqüência e a angulação do aparelho ( $r=$ 0,970) (Figura 1). 


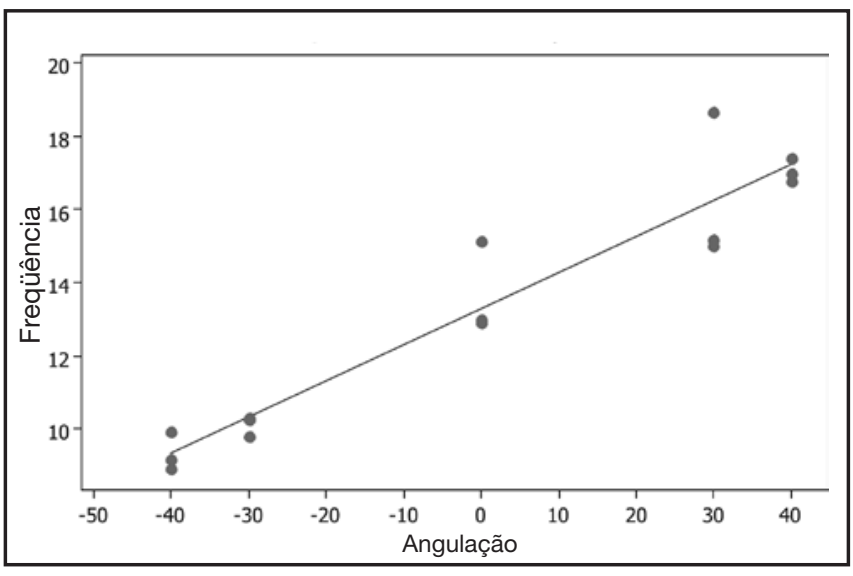

Figura 1 - Variação da Freqüência em Relação à Angulação

O mesmo foi constatado para a pressão em relação à angulação utilizada ( $r=0,942)$ (Figura 2). As pressões expiratórias e as freqüências de oscilação apresentaram-se significativamente diferentes nas angulações negativas se comparados aos ângulos positivos.

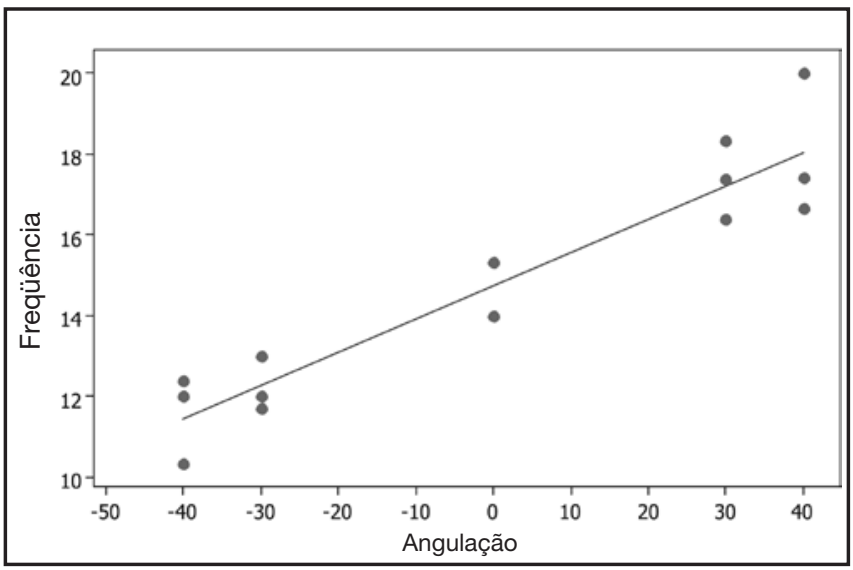

Figura 2 - Variação da Pressão em Relação a Angulação

\section{DISCUSSÃO}

Usando um modelo experimental in vitro, a relação entre a pressão expiratória e a freqüência de oscilação foi avaliada em relação à inclinação do aparelho em questão. Os resultados deste estudo indicaram que a inclinação positiva $\left(30^{\circ}\right.$ e $\left.40^{\circ}\right)$ associadas as taxas de fluxos aéreos mais altos (50 e $60 \mathrm{~L} / \mathrm{min}$ ) resultram em aumento na pressão e na freqüência de oscilação. O modelo in vitro foi escolhido para controle da variabilidade do fluxo, pois esta é extremamente difícil em modelos in vivo.

Este estudo confirmou os resultados descritos por outros autores ${ }^{11}$, que também demonstraram a relação entre estas variáveis, porém com fluxos aéreos bem menores. Os valores de fluxo aéreos utilizados neste estudo foram baseados nos valores fisiológicos encontrados durante a expiração lenta não forçada. Em estudo similar a este, as propriedades viscoelásticas da secreção de pacientes com fibrose cística, foi satisfatoriamente alterada durante a utilização de um fluxo aéreo médio de $60 \mathrm{~L} / \mathrm{min}$ o qual, gerou uma freqüência de oscilação em torno de $17 \mathrm{~Hz}^{1}$. A variabilidade encontrada nos valores da pressão expiratória e da freqüência de oscilação no presente estudo foi similar aos anteriores ${ }^{11,12}$. Apesar de serem utilizados maiores fluxos as pressões expiratórias alcançadas não foram altas em todas as angulações, como os valores encontrados em estudo similar ${ }^{11}$. Diz-se que pressões superiores a $20 \mathrm{cmH}_{2} \mathrm{O}$ podem conduzir ao barotrauma e maior risco de aprisionamento aéreo. Assim, pelo fato do Shaker não produzir pressões superiores a $18 \mathrm{cmH}_{2} \mathrm{O}$ estes riscos são reduzidos, efeito extremamente desejável em pacientes ventilados artificialmente.

Na prática fisioterápica raramente os ângulos e fluxos dos aparelhos de oscilação oral de alta freqüência são mensurados. Assim o entendimento, de que o ângulo positivo associado a maior taxa de fluxo resulta em aumento na freqüência de oscilação e na pressão expiratória farão com que o terapeuta haja com maior cautela ao implementar esta terapia em pacientes considerados críticos.

\section{CONCLUSÃO}

Neste estudo foi verificado o efeito da variação do fluxo e da angulação do aparelho de oscilação oral de alta freqüência em relação às pressões expiratórias e freqüências obtidas. Os resultados demonstraram que o aparelho obtém maior freqüência de oscilação e menor pressão com angulações positivas $\left(30^{\circ}\right.$ e $\left.40^{\circ}\right)$. Estes novos dados sugerem um maior entendimento à cerca do funcionamento deste aparelho. Sendo assim, relevante para o terapeuta respiratório que o utiliza em unidades de terapia intensiva associado ao ventilador mecânico, como recurso coadjuvante às técnicas manuais de desobstrução brônquica.

Pesquisas futuras devem ser realizadas para avaliar a relação destas variáveis in vivo com o ventilador mecânico. 


\section{REFERÊNCIAS}

01. Fink BJ, Mahlmeister JM - High-frequency oscillation of the airway and chest wall. Respir Care, 2001;47:797-807.

02. Weiner P, Zamir D, Waizman J et al - Physiotherapy in chronic obstructive pulmonary disease: oscillatory breathing with flutter VRP1. Harefuah, 1996;131:14-17.

03. Darbee JC, Ohtake PJ, Grant BJ et al - Physiologic Evidence for the efficacy of positive expiratory pressure as an airway clearance technique in patients with cystic fibrosis. Phys Ther, 2004;84:524-537.

04. Dasgupta B, Tomkiewicz RP, Boyd WA et al - Effects of combined treatment with rhDNase and airflow oscillations on spinnability of cystic fibrosis sputum in vitro. Pediatr Pulmonol, 1995;20:78-82.

05. Chang HK, Weber ME, King M - Mucus transport by high-frequency nonsymmetrical oscillatory airflow. J Appl Physiol, 1988;65:1203-1209.

06. Leru P, Bistriceanu G, Ibraim E et al - Flutter-VRP1 Desitin--a new phy- siotherapeutic device for the treatment of chronic obstructive bronchitis. Rom J Intern Med, 1994;32:315-320.

07. King M, Zidulka A, Phillips DM - Traqueal mucus clearance in high-frequency oscillation: effect of peak flow rate bias. Eur Respir J, 1990;3:613.

08. Wolkove N, Kamel H, Rotaple M et al - Use of a mucus clearance device enhances the bronchodilator response in patients with stable COPD. Chest, 2002;121:702-707.

09. Adair CG, Gorman SP, Feron BM et al - Implications of the endotraqueal tube biofilm for ventilator-associated pneumonia. Intensive Care Med, 1999;25:1072-1076.

10. Corona A, Raimondi F - Prevention of nosocomial infection in the ICU setting. Minerva Anesthesiol, 2004;70:329-337.

11. Brooks D, Newbold E, Kozar LF et al - The flutter device and expiratory pressures. J Cardiopulm Rehabil, 2002;22:53-57.

12. Lindemann $\mathrm{H}$ - The value of physical therapy with VRP1-Desitin (Flutter). Pneumologie, 1992;46:626-630. 